\title{
Single-mode approximation and effective Chern-Simons theories for quantum Hall systems
}

\author{
K. Shizuya \\ Yukawa Institute for Theoretical Physics \\ Kyoto University, Kyoto 606-8502, Japan
}

\begin{abstract}
A unified description of elementary and collective excitations in quantum Hall systems is presented within the single-mode approximation (SMA) framework, with emphasis on revealing an intimate link with Chern-Simons theories. It is shown that for a wide class of quantum Hall systems the SMA in general yields, as an effective theory, a variant of the bosonic Chern-Simons theory. For single-layer systems the effective theory agrees with the standard Chern-Simons theory at long wavelengths whereas substantial deviations arise for collective excitations in bilayer systems. It is suggested, in particular, that Hall-drag experiments would be a good place to detect out-ofphase collective excitations inherent to bilayer systems. It is also shown that the intra-Landau-level modes bear a similarity in structure (though not in scale) to the inter-Landau-level modes, and its implications on the composite-fermion and composite-boson theories are discussed.
\end{abstract}

\section{INTRODUCTION}

The early theoretical study of the fractional quantum Hall effect ${ }^{1}$ (FQHE), based on Laughlin's wave functions, ${ }_{2}^{2}$ revealed that incompressibility is the key character of the quantum Hall states. This observation then evolved ${ }^{3.4}$ into new pictures of the FQHE in term of electron-flux composites, the composite bosons or composite fermions. There the fractional quantum Hall states are either visualized as charged superfluids with Bose-condensed composite bosons in zero magnetic field or mapped to integer-quantum-Hall states of composite fermions in a reduced magnetic field. Chern-Simons (CS) theories, both bosonic ${ }^{5.6 .7}$ and fermionic, ${ }^{8.9 .10}$ realize these composite-particle descriptions of the FQHE by an expansion around mean field.

There is apparently another stream in the theory of the FQHE, that enforces the importance of projecting the dynamics onto the (lowest) Landau level, a key point emphasized in the wave-function approach. There are several approximation schemes of this sort, 11.12 .13 .14 .15 .16 .17 .18 both perturbative and nonperturbative. The single-mode approximation (SMA), in particular, is a general method to study collective excitations in liquid states. The SMA equipped with such projection, developed by Girvin, MacDonald and Platzman, $\stackrel{13}{=}$ has proved to be a powerful nonperturbative means to explore quantum Hall systems, even better suited than the well-known case of liquid Helium.

The CS and SMA approaches, though equally successful in revealing various aspects of the FQHE, appear rather independent. Appealing physical pictures in CS approaches should be contrasted with the generality in formalism (enforcing Landau-level projection, sum rules, etc.) of the SMA theory. In certain cases they even lead to subtle differences $\underline{17}$

The purpose of this paper is to present a unified description of elementary and collective excitations in quantum Hall systems within the SMA theory and to uncover an intimate link between the SMA and CS theories. In particular, we show that for a wide class of quantum Hall systems the SMA in general yields, as an effective theory, a variant of the bosonic CS theory. For single-layer systems the effective theory agrees with the standard CS theory at long wavelengths whereas substantial deviations arise for collective excitations in bilayer systems. Such a link between the SMA theory and the compositeboson theory was earlier noticed indirectly through the response of quantum Hall states $\frac{19}{19}$ In this paper we establish it directly within the SMA theory, generalize it to higher-multipole excitations in quantum Hall systems and discuss its implications on the composite-fermion theory as well as the composite-boson theory.

We present the basic formalism by studying, for singlelayer systems, the cyclotron modes and collective modes in Sec. II and III, respectively. It will be seen that the intra-Landau-level modes bear a similarity in structure (though not in scale) to the inter-Landau-level modes. In Sec. IV we examine bilayer systems. Section VI is devoted to a summary and discussion.

\section{EFFECTIVE THEORY - FORMALISM}

Consider a quantum Hall system described by the Hamiltonian

$H_{0}=\int d^{2} \mathbf{x} \Psi^{\dagger}(x) \frac{1}{2 M}\left(\mathbf{p}+e \mathbf{A}^{B}\right)^{2} \Psi(x)+H^{\mathrm{Coul}}$,

where $\mathbf{A}^{B}=B\left(-x_{2}, 0\right)$ supplies a uniform magnetic field $B_{z}=B>0$ normal to the $\mathbf{x}=\left(x_{1}, x_{2}\right)$ sample plane. For definiteness we take a single-layer system with spinpolarized electrons; the spin and layer degrees of freedom are readily included.

Out of the electron field $\Psi(x)$ one can form a number of charge operators. Actually there are an infinite number of them reflecting the Landau levels of the electron. This is made explicit by expanding the electron field $\Psi(\mathbf{x}, t)=$ $\sum_{N}\langle\mathbf{x} \mid N\rangle \Psi_{n}\left(y_{0}, t\right)$ in terms of the Landau levels $|N\rangle=$ $\left|n, y_{0}\right\rangle$ of a freely orbiting electron of energy $\omega_{c}\left(n+\frac{1}{2}\right)$ 
with $n=0,1,2, \cdots$, and $y_{0}=\ell^{2} p_{x_{1}}$, where $\omega_{c} \equiv e B / M$ and $\ell \equiv 1 / \sqrt{e B}$; we frequently set $\ell \rightarrow 1$ below. The charge operator $\rho_{\mathbf{p}}=\int d^{2} \mathbf{x} e^{-i \mathbf{p} \cdot \mathbf{x}} \Psi^{\dagger} \Psi$ then reads ${ }^{12,13,20}$

$$
\begin{aligned}
\rho_{\mathbf{p}} & =\sum_{m, n=0}^{\infty} F^{m n}(\mathbf{p}) R_{\mathbf{p}}^{(m n)} \\
R_{\mathbf{p}}^{(m n)} & =\int d y_{0} \Psi_{m}^{\dagger}\left(y_{0}, t\right) e^{-\frac{1}{4} \ell^{2} \mathbf{p}^{2}} e^{-i \mathbf{p} \cdot \mathbf{r}} \Psi_{n}\left(y_{0}, t\right)
\end{aligned}
$$

Here $\mathbf{r} \equiv\left(r_{1}, r_{2}\right)=\left(i \ell^{2} \partial / \partial y_{0}, y_{0}\right)$ stands for the center coordinates with uncertainty $\left[r_{1}, r_{2}\right]=i \ell^{2}$, and

$$
F^{m n}(\mathbf{p})=\left\langle m\left|e^{-i(p / \sqrt{2}) Z^{\dagger}} e^{-i\left(p^{\dagger} / \sqrt{2}\right) Z}\right| n\right\rangle
$$

with $\left[Z, Z^{\dagger}\right]=1$ and $Z^{\dagger} Z|n\rangle=n|n\rangle ; p=p_{2}+i p_{1}$. In particular, $F^{m n}(\mathbf{p})=\sqrt{n ! / m !}(-i p / \sqrt{2})^{m-n} L_{n}^{(m-n)}\left(\mathbf{p}^{2} / 2\right)$ for $m \geq n ; F^{00}(\mathbf{p})=1, F^{n 0}(\mathbf{p})=(-i p / \sqrt{2})^{n} / \sqrt{n !}$, and $F^{0 n}(\mathbf{p})=\left(-i p^{\dagger} / \sqrt{2}\right)^{n} / \sqrt{n !}$. The fields $\Psi_{n}\left(y_{0}, t\right)$ obey the canonical anticommutation relations $\left\{\Psi_{m}\left(y_{0}, t\right), \Psi_{n}^{\dagger}\left(y_{0}^{\prime}, t\right)\right\}=\delta_{m n} \delta\left(y_{0}-y_{0}^{\prime}\right)$. The underlying Landau-level structure is now encoded in the $U_{\infty}$ or $W_{\infty}$ algebra 13 obeyed by the charges $R_{\mathbf{p}}^{(m n)}$ :

$$
\left[R_{\mathbf{k}}^{\left(m m^{\prime}\right)}, R_{\mathbf{p}}^{\left(n n^{\prime}\right)}\right]=\delta^{m^{\prime} n} e^{\frac{1}{2} k^{\dagger} p} R_{\mathbf{k}+\mathbf{p}}^{\left(m n^{\prime}\right)}-\delta^{n^{\prime} m} e^{\frac{1}{2} p^{\dagger} k} R_{\mathbf{k}+\mathbf{p}}^{\left(n m^{\prime}\right)} .
$$

The charges $\rho_{\mathbf{p}}^{(m n)}=F^{m n}(\mathbf{p}) R_{\mathbf{p}}^{(m n)}$ generate interand intra-Landau-level excitations for $m \neq n$ and $m=n$, respectively. Our task in this section is to study such excitations, both elementary and collective, over a quantum Hall state by means of the single-mode approximation ${ }^{13.15}$ (SMA). Let $|G\rangle$ denote an exact quantum Hall (i.e., incompressible) ground state of the Hamiltonian $H_{0}$ with uniform density $\rho_{0}$. In the SMA one represents the excitation modes as $\left|\phi_{\mathbf{k}}^{\alpha}\right\rangle \sim \rho_{\mathbf{k}}^{\alpha}|G\rangle$ with $\alpha=(m, n)$, and regards their normalization

$$
s^{\alpha}(\mathbf{k})=\left(1 / N_{e}\right)\left\langle G\left|\left(\rho_{\mathbf{k}}^{\alpha}\right)^{\dagger} \rho_{\mathbf{k}}^{\alpha}\right| G\right\rangle
$$

called the static structure factors, as the basic quantity; $N_{e}$ stands for the total electron number. Saturating the $f$-sum rule or the oscillator strength

$$
f^{\alpha}(\mathbf{k})=\left(1 / N_{e}\right)\left\langle G\left|\left(\rho_{\mathbf{k}}^{\alpha}\right)^{\dagger}\left[H_{0}, \rho_{\mathbf{k}}^{\alpha}\right]\right| G\right\rangle
$$

calculable by use of the charge algebra (2.4), with the single mode $\left|\phi_{\mathbf{k}}^{\alpha}\right\rangle$ then yields the SMA excitation spectrum $\epsilon_{\mathbf{k}}^{\alpha}=f^{\alpha}(\mathbf{k}) / s^{\alpha}(\mathbf{k})$. The spectrum is determined once $s^{\alpha}(\mathbf{k})$ is known.

The SMA sets up one-to-one correspondence between the excitations and Landau-level charges $\rho_{\mathbf{p}}^{(m n)}$. The assumption of single-mode dominance is far from obvious but exact-diagonalization studies of small systems generally suggest that it is a good approximation for the lowest-lying collective modes 13 We therefore pursue the SMA here.
Let us now try to construct an effective theory realizing the SMA description of excitations. The correspondence noted above suggests us to use the technique ${ }^{18}$ of nonlinear realization of the $W_{\infty}$ algebra for this purpose. First let $\Psi_{0}^{\mathrm{cl}}\left(y_{0}, t\right)$ denote a classical configuration or the ground-state configuration, with pertinent correlations characterized by (a set of) static structure factors $s^{\alpha}(\mathbf{k})$. We then write the electron field $\Psi$ in the form of a small variation in phase from $\Psi^{\mathrm{cl}}$,

$$
\Psi \sim \exp \left[-i \sum_{\alpha} \sum_{\mathbf{p}} \theta_{-\mathbf{p}}^{\alpha} T_{\mathbf{p}}^{\alpha}\right] \Psi^{\mathrm{cl}}
$$

Here $\theta_{-\mathbf{p}}^{\alpha}=\theta_{-\mathbf{p}}^{(m n)}$ with $\left(\theta_{\mathbf{p}}^{(m n)}\right)^{\dagger}=\theta_{-\mathbf{p}}^{(n m)}$ stand for local phase variations; $T_{\mathbf{p}}^{\alpha}=F^{m n}(\mathbf{p}) e^{-\frac{1}{4} \mathbf{p}^{2}} e^{-i \mathbf{p} \cdot \mathbf{r}}$. Rewriting the Lagrangian in favor of $\Psi^{\mathrm{cl}}$ and $\theta$, and replacing the products of $\left(\Psi^{\mathrm{cl}}\right)^{\dagger}$ and $\Psi^{\mathrm{cl}}$ by the structure factors then yields an effective Lagrangian for the excitation modes $\theta_{\mathbf{p}}^{\alpha}$.

For such transcription it is convenient to express Eq. (2.7) in operator form

$$
\begin{aligned}
& \Psi=\mathcal{P}^{-1} \Psi^{\mathrm{cl}} \mathcal{P} \\
& \mathcal{P}=e^{-i \theta \cdot \rho^{\mathrm{cl}}}, \theta \cdot \rho^{\mathrm{cl}} \equiv \sum_{\mathbf{p}} \sum_{\alpha} \theta_{-\mathbf{p}}^{\alpha}\left(\rho_{\mathbf{p}}^{\alpha}\right)^{\mathrm{cl}}, \text { etc. }
\end{aligned}
$$

Here $\left(\rho_{\mathbf{p}}^{\alpha}\right)^{\mathrm{cl}}$ stand for $\rho_{\mathbf{p}}^{\alpha}$ with $\Psi$ replaced by $\Psi^{\mathrm{cl}}$, and obey the same charge algebra as $\rho_{\mathbf{p}}^{\alpha}$. Repeated use of the algebra then enables one to express $\rho_{\mathbf{p}}^{\alpha}=\mathcal{P}\left(\rho_{\mathbf{p}}^{\alpha}\right)^{\mathrm{cl}} \mathcal{P}^{-1}$ in powers of $\left(\rho_{\mathbf{p}}^{\alpha}\right)^{\mathrm{cl}}$.

Substituting Eq. (2.7) into the Lagrangian $L=$ $\int d^{2} \mathbf{x} \Psi^{\dagger} i \partial_{t} \Psi-H_{0}$ yields a Berry's phase ${ }^{21}$ contribution $\left(\Psi^{\mathrm{cl}}\right)^{\dagger} e^{i \theta \cdot T}\left(i \partial_{t} e^{-i \theta \cdot T}\right) \Psi^{\mathrm{cl}}$, which, by use of the charge algebra (2.4), is cast in the compact form

$$
e^{i \theta \cdot \rho^{\mathrm{cl}}} i \partial_{t} e^{-i \theta \cdot \rho^{\mathrm{cl}}}=\dot{\theta} \cdot \rho^{\mathrm{cl}}+\frac{i}{2}\left[\theta \cdot \rho^{\mathrm{cl}}, \dot{\theta} \cdot \rho^{\mathrm{cl}}\right]+\cdots,
$$

where $\partial_{t}$ acts on $\theta ; \dot{\theta}=\partial_{t} \theta$. The Lagrangian then reads

$$
L=e^{i \theta \cdot \rho^{\mathrm{cl}}}\left(i \partial_{t}-H_{0}^{\mathrm{cl}}\right) e^{-i \theta \cdot \rho^{\mathrm{cl}}}
$$

where $H_{0}^{\mathrm{cl}}$ stands for $H_{0}$ with $\Psi \rightarrow \Psi^{\mathrm{cl}}$. We shall from now on exclusively handle $\Psi^{\mathrm{cl}}$ and $\left(\rho_{\mathbf{p}}^{\alpha}\right)^{\mathrm{cl}}$, and suppress the suffix "cl" unless confusion arises.

Suppose now that $|G\rangle$ consists predominantly of the lowest-Landau-level $(n=0)$ components, with the filling factor $\nu=2 \pi \ell^{2} \rho_{0}<1$. Taking the expectation value of Eq. (2.10) then yields the effective Lagrangian

$$
\langle L\rangle=\rho_{0} \sum_{\mathbf{k}} \sum_{n=1}^{\infty} \theta_{\mathbf{k}}^{(0 n)}\left[i s^{(n 0)}(\mathbf{k}) \partial_{t}+f^{(n 0)}(\mathbf{k})\right] \theta_{-\mathbf{k}}^{(n 0)}
$$

to $O\left(\theta^{2}\right)$, apart from total divergences; $\langle\cdots\rangle \equiv\langle G|\cdots| G\rangle$ for short. Here $s^{(n 0)}(\mathbf{k})=\left(1 / N_{e}\right)\left\langle G\left|\rho_{-\mathbf{k}}^{(0 n)} \rho_{\mathbf{k}}^{(n 0)}\right| G\right\rangle$ are the structure factors and $f^{(n 0)}(\mathbf{k})$ are the associated oscillator strengths; $\rho_{0}=N_{e} / \Omega$ with the total area $\Omega$. Actually, noting that $\left\langle R_{\mathbf{k}}^{(00)}\right\rangle=\rho_{0} \delta_{\mathbf{k}, \mathbf{0}}$, and $\left\langle R_{\mathbf{k}}^{(0 n)} R_{\mathbf{p}}^{(n 0)}\right\rangle=$ 
$\rho_{0} e^{-\frac{1}{2} \mathbf{k}^{2}} \delta_{\mathbf{k}+\mathbf{p}, \mathbf{0}}$ with $\delta_{\mathbf{k}, \mathbf{0}}=(2 \pi)^{2} \delta^{2}(\mathbf{k})$, one finds that

$$
\begin{aligned}
& s^{(n 0)}(\mathbf{k})=(1 / n !)\left(\ell^{2} \mathbf{k}^{2} / 2\right)^{n} e^{-\frac{1}{2} \ell^{2} \mathbf{k}^{2}}, \\
& f^{(n 0)}(\mathbf{k})=n \omega_{c} s^{(n 0)}(\mathbf{k})+O\left(H^{\text {Coul }}\right) .
\end{aligned}
$$

for $n \geq 1$. The Lagrangian (2.10) tells us that $\theta^{(n 0)}$ are canonically conjugate to $\theta^{(0 n)}=\left(\theta^{(n 0)}\right)^{\dagger}$ for $n \geq$ 1. They describe the $(0 \rightarrow n)$ cyclotron modes with the spectrum $\epsilon_{\mathbf{k}}^{(n 0)}=f^{(n 0)}(\mathbf{k}) / s^{(n 0)}(\mathbf{k}) \approx n \omega_{c}$; physically they represent neutral exciton excitations ${ }^{11}$ formed of a hole in the zero-th level and an electron in the $n$th level. Note here that the intra-Landau-level mode $\theta^{(00)}$, which finds no canonical conjugate, requires a separate analysis, which will be given in Sec. III.

Let us now generalize the framework by including coupling to weak external electromagnetic potentials $A_{\mu}(x)=\left(A_{1}, A_{2}, A_{0}\right)$. Replacing the kinetic term in Eq. (2.1) with $(1 / 2 M)\left(\mathbf{p}+e \mathbf{A}^{B}+e \mathbf{A}\right)^{2}+e A_{0}$ yields the Hamiltonian $H=H_{0}+U$ with

$$
\begin{aligned}
U= & \sum_{\mathbf{p}} \sum_{m, n} U_{-\mathbf{p}}^{m n} R_{\mathbf{p}}^{(m n)} \\
U_{-\mathbf{p}}^{m n}= & i \ell \omega_{c}\left\{A_{-\mathbf{p}} \sqrt{m} F^{m-1, n}(\mathbf{p})-A_{-\mathbf{p}}^{\dagger} \sqrt{n} F^{m, n-1}(\mathbf{p})\right\} \\
& +\tilde{\chi}_{-\mathbf{p}} F^{m n}(\mathbf{p}),
\end{aligned}
$$

where $A(x)=\left(A_{2}+i A_{1}\right) / \sqrt{2}$ and $A^{\dagger}=\left(A_{2}-i A_{1}\right) / \sqrt{2}$; $\tilde{\chi}=A_{0}+(1 / 2 M)\left(A_{12}+A_{k}^{2}\right), A_{12}=\partial_{1} A_{2}-\partial_{2} A_{1} ; \tilde{\chi}_{\mathbf{p}}, A_{\mathbf{p}}$, etc., stand for the Fourier transforms (with obvious time dependence suppressed); the electric charge $e$ has been suppressed by rescaling $e A_{\mu} \rightarrow A_{\mu}$.

The $U$ turns into the effective interaction $\mathcal{P} U \mathcal{P}^{-1}=$ $U+i[\theta \cdot \rho, U]+\cdots$. It suffices to calculate only the $O(A \theta)$ coupling to derive the electromagnetic response to $O\left(A^{2}\right)$ eventually. To simplify the result it is convenient to express $\theta^{(n 0)}$ in terms of real fields $\eta^{(n)}(x)$ and $\xi^{(n)}(x)$,

$$
\begin{aligned}
& \gamma_{\mathbf{k}} \theta_{\mathbf{k}}^{(n 0)}=\xi_{\mathbf{k}}^{(n)}+i \eta_{\mathbf{k}}^{(n)}, \\
& \gamma_{\mathbf{k}}=\frac{1}{\sqrt{n !}}\left(\frac{1}{2} \ell^{2} \mathbf{k}^{2}\right)^{(n-1) / 2} e^{-\frac{1}{4} \ell^{2} \mathbf{k}^{2}}
\end{aligned}
$$

and to pass from the Fourier space to real $\mathbf{x}$ space. The effective Lagrangian to $O(A \theta)$ is cast in the form $\langle L\rangle=$ $\int d^{2} \mathbf{x}\left(\mathcal{L}_{A}+\mathcal{L}_{A \theta}\right)$ with

$$
\begin{aligned}
\mathcal{L}_{A}= & -\rho_{0}\left(A_{0}+\frac{1}{2 M} A_{j}^{2}\right) \\
\mathcal{L}_{\theta A}= & \rho_{0}[2 \eta s(\dot{\xi}-\gamma \chi)-(\eta f \eta+\xi f \xi) \\
& \left.\quad-\kappa \ell^{2} \xi \gamma \partial_{j} A_{j}+\kappa \ell^{2} \eta \gamma A_{12}\right] .
\end{aligned}
$$

Here $\chi=A_{0}+(1 / 2 M) A_{12}, \kappa=n \omega_{c}$ and

$$
\begin{aligned}
& s \equiv s^{(n 0)}(\mathbf{k}) / \gamma^{2}=\ell^{2} \mathbf{k}^{2} / 2, \\
& f \equiv f^{(n 0)}(\mathbf{k}) / \gamma^{2}=n \omega_{c} \ell^{2} \mathbf{k}^{2} / 2,
\end{aligned}
$$

with $\mathbf{k}=-i \nabla$ understood and $\gamma=\gamma_{\mathbf{k}}$. The $\mathcal{L}_{A}$ comes from $-\langle U\rangle$ and $\mathcal{L}_{\theta A}$ involves the $O\left(\theta^{2}\right)$ term of Eq. (2.11) as well. For conciseness explicit reference to the mode index $n$ has been suppressed in the above; remember that a summation over all modes is implied in $\mathcal{L}_{\theta A}$.

It is a straightforward but strenuous task to calculate the $O\left(H^{\text {Coul }}\right)$ portion 15 of $f^{(n 0)}(\mathbf{k})$, left out from $f$ in Eq. (2.17). An alternative and simpler way, that works to extract the leading long-wavelength part of it, is to first linearize the Coulomb interaction $\frac{1}{2} \sum_{\mathbf{k}} V_{\mathbf{k}} \rho_{-\mathbf{k}} \rho_{\mathbf{k}}$ by use of a Hubbard-Stratonovich field $\phi$. The net effect is to replace $\chi$ by $\chi+\phi$ in Eq. (2.16). Eliminating $\phi$ then yields the effective interaction

$$
\mathcal{L}^{\text {Coul }}=-2 \rho_{0}^{2} \eta s \gamma V_{\mathbf{k}} s \gamma \eta
$$

The $(0 \rightarrow n)$ exciton modes are now described by the effective Lagrangian $\mathcal{L}_{A}+\mathcal{L}_{A \theta}+\mathcal{L}^{\text {Coul }}$. Let us rewrite it in a more suggestive form. We consider the $(0 \rightarrow 1)$ mode first and set $\gamma \rightarrow 1$. For generality and later use we leave the normalization of the reduced factors, $\hat{s} \equiv s /\left(\ell^{2} \mathbf{k}^{2} / 2\right)$ and $\hat{f} \equiv f /\left(\ell^{2} \mathbf{k}^{2} / 2\right)$, arbitrary and denote the spectrum $\epsilon=f / s=\hat{f} / \hat{s}$ [although $\hat{s}=1$ and $\hat{f}=\epsilon=n \omega_{c}$ in the present case]. Let us combine the $\xi^{2}, \xi \partial_{j} A_{j}$ and $\eta A_{12}$ terms to form a complete square so that

$$
\begin{aligned}
& \mathcal{L}_{A \theta}+\mathcal{L}^{\text {Coul }} \\
& =r\left(\dot{\xi}-A_{0}\right)-\frac{1}{2} \rho_{0} \ell^{2} \hat{f}\left(\partial_{j} \xi-\frac{\kappa}{\hat{f}} A_{j}+\frac{\delta}{2 \rho_{0} s} \epsilon_{j k} \partial_{k} r\right)^{2} \\
& -\frac{1}{2} r\left(V_{\mathbf{k}}+\triangle\right) r+\frac{1}{2} \rho_{0} \ell^{2} \kappa^{2} A_{j} \frac{1}{\hat{f}} A_{j},
\end{aligned}
$$

where we have set $r=2 \rho_{0} s \eta$ and $\triangle=\left(1-\delta^{2}\right) \epsilon /\left(2 \rho_{0} s\right)$ with $\delta=1-s \omega_{c} / \kappa ; \epsilon_{12}=-\epsilon_{21}=1$.

Note that for gauge invariance it is necessary to have $\kappa / \hat{f}=1$ or $f=\frac{1}{2} \kappa \ell^{2} \mathbf{k}^{2}+O\left(\mathbf{k}^{4}\right)$, which is satisfied in the present case. The last term in Eq. (2.19), though spoiling gauge invariance, is harmless. For the $(0 \rightarrow n)$ mode it reads $\propto \frac{1}{2} \rho_{0} \ell^{2} n \omega_{c} A_{j}\left(\gamma_{\mathbf{k}}\right)^{2} A_{j}$, which, when summed over $n=1,2, \cdots$, amounts to $\frac{1}{2} \rho_{0} \ell^{2} \omega_{c} A_{j}^{2}$. Thus this term combines with the $A^{2}$ term in $\mathcal{L}_{A}$ to vanish exactly.

The $(1 / s) \epsilon_{j k} \partial_{k} r$ term in Eq. (2.19) can be disentangled by use of a vector field $c_{\mu}$, thereby yielding an effective Chern-Simons theory described by the Lagrangian

$$
\begin{aligned}
\mathcal{L}_{c}= & r\left(\dot{\xi}-A_{0}-c_{0}\right)-\frac{1}{2} \rho_{0} \ell^{2} \hat{s} \epsilon\left(\partial_{j} \xi-A_{j}-c_{j}\right)^{2} \\
& -\frac{1}{2} r\left(V_{\mathbf{k}}+\triangle\right) r-\frac{1}{2} c_{\mu} \alpha \epsilon^{\mu \nu \rho} \partial_{\nu} c_{\rho}
\end{aligned}
$$

with $\alpha=\rho_{0} \ell^{2} \kappa /(\epsilon \delta) ; \epsilon^{012}=1$. [One may practically set $\delta=1+O\left(\ell^{2} \mathbf{k}^{2}\right) \rightarrow 1$ and $\alpha \rightarrow \rho_{0} \ell^{2}=\nu /(2 \pi)$ here. For the full Lagrangian, include in $\mathcal{L}_{c}$ the $-\rho_{0} A_{0}$ term coming from $\mathcal{L}_{A}$.] The equivalence of $\mathcal{L}_{c}$ to $\mathcal{L}_{A \theta}+\mathcal{L}^{\text {Coul }}$ is immediately seen in the Coulomb gauge $\partial_{k} c_{k}=0$.

For the Laughlin sequence $\nu=1 / 3,1 / 5, \cdots$ this effective theory precisely agrees with the standard CS theor $\mathrm{y}^{\underline{5}}$ with the composite-boson field $\phi_{\mathrm{cb}}(x)=\sqrt{\rho_{0}+r} e^{-i \xi}$ expanded to second order in $r$ and $\xi$ around the mean field, except for the $\triangle \approx \epsilon \omega_{c} /\left(\rho_{0} \kappa\right)$ term which in the latter 
reads $\ell^{2} \mathbf{k}^{2} \omega_{c} /\left(4 \rho_{0}\right)$. This $\triangle$ term is less important than the Coulomb term $V_{\mathbf{k}} \sim 1 /|\mathbf{k}|$ at long wavelengths.

The filling factor $\nu<1$ is not fixed within the present approach. The effective CS theory (2.20) makes sense for general $\nu$ as long as (i) the state $|G\rangle$ is incompressible and (ii) the SMA is applicable (approximately).

It is possible to cast the theory into an equivalent dualfield $^{6}$ form, suited for discussing the dynamics of vortices. We first use a vector field $j_{k}$ to linearize the complete square term in Eq. (2.19) so that $j_{k}\left(\partial_{k} \xi+\cdots\right)+$ $\left(1 / 2 \rho_{0}\right) j_{k}(1 / \hat{f}) j_{k}$ and then eliminate $\xi$. The resulting conservation law $\partial_{\mu} j_{\mu}=0$ with $j_{0} \equiv r$ allows one to set $j_{\mu}=\epsilon^{\mu \nu \lambda} \partial_{\nu} b_{\lambda}$ with a three-vector field $b_{\mu}=\left(b_{k}, b_{0}\right)$. Substituting this back into Eq. (2.19) then yields an equivalent theory described by the Lagrangian

$$
\begin{aligned}
\mathcal{L}_{b}= & -A_{\mu} \epsilon^{\mu \nu \rho} \partial_{\nu} b_{\rho}+\frac{1}{2 \rho_{0} \ell^{2}} b_{\mu} \frac{\delta}{\hat{s}} \epsilon^{\mu \nu \rho} \partial_{\nu} b_{\rho} \\
& +\frac{1}{2 \rho_{0} \ell^{2}} b_{k 0} \frac{1}{\hat{s} \epsilon} b_{k 0}-\frac{1}{2} b_{12}\left(V_{\mathbf{k}}+\triangle\right) b_{12}
\end{aligned}
$$

The third term is obtained with the aid of the formula

$$
j_{k} \epsilon_{k i} \partial_{i} j_{0}=-\frac{1}{2} b_{\mu} \nabla^{2} \epsilon^{\mu \nu \rho} \partial_{\nu} b_{\rho}+\text { total div. }
$$

From this $\mathcal{L}_{b}$ one can calculate an electromagnetic response of the form $S[A]=\int d^{3} x \mathcal{L}[A]$ with

$$
\begin{aligned}
\mathcal{L}[A]=\frac{1}{2}\{ & -A_{\mu} \delta \mathcal{D} \epsilon^{\mu \nu \rho} \partial_{\nu} A_{\rho}+A_{k 0} \frac{1}{\epsilon} \mathcal{D} A_{k 0} \\
& \left.-A_{12} \Sigma \mathcal{D} A_{12}\right\}, \\
\mathcal{D}= & \rho_{0} \ell^{2} \hat{s} \frac{\epsilon^{2}}{E_{\mathbf{k}}^{2}-\omega^{2}},
\end{aligned}
$$

where $\omega=i \partial_{t}$ and $\Sigma=\rho_{0} \ell^{2} \hat{s}\left(V_{\mathbf{k}}+\triangle\right)$, with the excitation spectrum given by

$$
E_{\mathbf{k}}^{2} \approx \epsilon^{2}+2 \rho_{0} \epsilon s V_{\mathbf{k}}
$$

Note that the Coulomb interaction hardly affects the long-wavelength response.

So far we have focused on the $(0 \rightarrow 1)$ cyclotron mode. For the higher modes one may simply set $A_{\mu} \rightarrow \gamma_{\mathbf{k}} A_{\mu}$, $V_{\mathbf{k}} \rightarrow \gamma_{\mathbf{k}} V_{\mathbf{k}} \gamma_{\mathbf{k}}$ and $\epsilon=\kappa \rightarrow n \omega_{c}$ in $\mathcal{L}_{c}, \mathcal{L}_{b}$ and $\mathcal{L}[A]$ in the above. It is seen that the $(0 \rightarrow n)$ exciton modes lead to the density-density response of $\langle\rho \rho\rangle \sim\left(\mathbf{k}^{2}\right)^{n}$ for $n \geq 1$. Thus only the $(0 \rightarrow 1)$ mode is dipole-active, i.e., sensitive to long-wavelength probes.

A link between the SMA theory and CS theories was noticed earlier ${ }^{19}$ for dipole-active excitations, indirectly through the response of quantum Hall systems. The advantage of the present approach, we remark, lies in establishing such a link directly within the SMA theory and, moreover, allowing one to study dipole-inactive (highermultipole) excitations equally well.

\section{INTRA-LANDAU-LEVEL EXCITATIONS}

In this section we introduce a variational principle for handling intra-Landau-level excitations. Experimentally collective excitations over the $\nu=1 / 3$ Laughlin state have been observed by inelastic light scattering 22 Correspondingly we here focus on excitations within the lowest Landau level, and denote the relevant $\theta_{\mathbf{k}}^{(00)}$ mode by $\bar{\theta}_{\mathbf{k}}$ and the charge operator $\rho_{\mathbf{p}}^{(00)}$ by $\bar{\rho}_{\mathbf{p}}$ for short; $\left(\bar{\theta}_{\mathbf{k}}\right)^{\dagger}=\bar{\theta}_{-\mathbf{k}}$ so that $\bar{\theta}(x)$ is a real field.

Let us denote the Lagrangian (2.10) anew as $L_{\theta}=$ $e^{i \theta \cdot \rho}\left(i \partial_{t}-H_{0}\right) e^{-i \theta \cdot \rho}$ with $\theta \cdot \rho \rightarrow \sum_{\mathbf{p}} \bar{\theta}_{-\mathbf{p}} \bar{\rho}_{\mathbf{p}}$ (and the superscript "cl" suppressed as before). This $L_{\theta}$ involves no canonical momentum conjugate to $\bar{\theta}(x)$. We try to supply it through a local variation in amplitude of the field. There are a number of ways to achieve this, but not all of them embody the SMA formalism. The variational ansatz we adopt is to consider

$$
L_{\lambda \theta}=\left\langle G\left|e^{\lambda \cdot \rho} L_{\theta} e^{\lambda \cdot \rho}\right| G\right\rangle=\langle G|\{\lambda \cdot \rho, \dot{\theta} \cdot \rho\}+\cdots| G\rangle,(3
$$

with $\lambda \cdot \rho=\sum_{\mathbf{k}} \lambda_{-\mathbf{k}} \bar{\rho}_{\mathbf{k}}$, where $\lambda_{\mathbf{k}}$ [or the real field $\lambda(x)$ ] denotes a local amplitude modulation.

Some care is needed here. The intra-Landau-level modes are governed by the Coulomb interaction and depend critically on the definition of the Landau levels. In order to derive a gauge-invariant result, it turns out necessary to define the Landau levels properly with the external potentials $A_{\mu}$ taken into account. We thus first make a unitary transformation $\Psi_{m}\left(y_{0}, t\right) \rightarrow \Psi_{n}^{\prime}\left(y_{0}, t\right)$ so that the one-body Hamiltonian becomes diagonal in Landau-level indices. Such a procedure of projection was developed earlier ${ }^{19}$ The one-body Hamiltonian thereby acquires terms of $O\left(A^{2}\right)$, which precisely agree with the electromagnetic response (2.23) due the cyclotron modes; thus, no explicit account of them is needed here. The relevant one-body Hamiltonian to $O(A)$ reads

$$
\bar{H}_{1}=\sum_{\mathbf{p}}\left(\frac{1}{2} \omega_{c} \delta_{\mathbf{p}, \mathbf{0}}+\chi_{\mathbf{p}}\right) \bar{\rho}_{-\mathbf{p}}
$$

with $\chi=A_{0}+(1 / 2 M) A_{12}$. Here $\bar{\rho}_{\mathbf{p}}$ are the charge operators defined in terms of the transformed field $\Psi_{n=0}^{\prime}\left(y_{0}, t\right)$; we suppress this specification because they obey the same algebra as $R_{\mathbf{p}}^{(00)}$ in Eq. (2.4).

An important consequence of projection is that the projected Coulomb interaction ${ }^{23}$

$$
\bar{H}^{\text {Coul }}=\frac{1}{2} \sum_{\mathbf{p}} V_{\mathbf{p}} \bar{\rho}_{-\mathbf{p}} \bar{\rho}_{\mathbf{p}}+\triangle H^{\mathrm{Coul}}
$$

acquires a field-dependent piece ${ }^{19}$ (which actually comes from the gauge invariance of the projected charges)

$$
\triangle H^{\text {Coul }}=\frac{1}{2} \sum_{\mathbf{p}, \mathbf{k}} V_{\mathbf{p}} u_{\mathbf{p}, \mathbf{k}}\left\{\bar{\rho}_{-\mathbf{p}}, \bar{\rho}_{\mathbf{p}-\mathbf{k}}\right\},
$$


where

$$
\begin{aligned}
u_{\mathbf{p}, \mathbf{k}}=i[ & \mathbf{p} \times \mathbf{A}-\frac{1}{4}\{\mathbf{p} \cdot \mathbf{k}(\mathbf{p} \times \mathbf{A})+(\mathbf{p} \times \mathbf{k}) \mathbf{p} \cdot \mathbf{A}\} \\
& \left.-\frac{1}{2} \mathbf{p} \cdot \mathbf{k}(\mathbf{k} \times \mathbf{A})+O\left(\mathbf{p}^{3} \mathbf{k}^{2} \mathbf{A}\right)\right]
\end{aligned}
$$

apart from terms of $O\left(1 / \omega_{c}\right) ; \mathbf{p} \times \mathbf{A}=\epsilon_{i j} p_{i}\left(A_{j}\right)_{\mathbf{k}}$, $\mathbf{p} \cdot \mathbf{A}=p_{i}\left(A_{i}\right)_{\mathbf{k}}$, etc. In Eq. (3.4) we have retained only $O(A)$ corrections, relevant to our discussion below.

Let us first turn $A_{\mu}$ off. Then the ground state $|G\rangle$ is an eigenstate of the Coulomb interaction, $H_{0}|G\rangle=E_{0}|G\rangle$ with $H_{0}=\bar{H}^{\text {Coul }}+$ quenched kinetic terms. We set $E_{0}=$ 0 by shifting the zero of energy. This is an important step since terms like $\left\langle\left\{\lambda \cdot \rho, H_{0}\right\}\right\rangle \sim \lambda_{\mathbf{k}=\mathbf{0}}$, which drive a constant shift in $\lambda$, thereby vanish. [The necessity of this shift in energy is consistent with the fact that the oscillator strength $f(\mathbf{k})$ involves only excitation energies measured relative to $E_{0}$.] The effective Lagrangian then takes the form $L_{\lambda \theta}=\int d^{2} \mathbf{x} \mathcal{L}_{0}$ with

$$
\mathcal{L}_{0}=\rho_{0}[2 \lambda \bar{s} \dot{\bar{\theta}}-(\lambda \bar{f} \lambda+\bar{\theta} \bar{f} \bar{\theta})] .
$$

This shows that $\lambda$ serves as a canonical conjugate of $\bar{\theta}$ and that $\bar{\theta}$ describes excitations with the spectrum $\epsilon=\bar{f} / \bar{s}$.

In general, the intra-Landau-level mode is dipole-inactive 13 and the structure factor $\bar{s}(\mathbf{k})=$ $\left(1 / N_{e}\right)\left\langle\bar{\rho}_{-\mathbf{k}} \bar{\rho}_{\mathbf{k}}\right\rangle$ starts with $O\left(\mathbf{k}^{4}\right)$,

$$
\bar{s}(\mathbf{k})=\frac{1}{2} c\left(\ell^{2} \mathbf{k}^{2}\right)^{2}+O\left(|\mathbf{k}|^{6}\right) .
$$

For the Laughlin wave functions

$$
c=(1-\nu) /(4 \nu)
$$

where $\nu=2 \pi \ell^{2} \rho_{0}$. The oscillator strength ${ }^{13}$

$$
\begin{aligned}
\bar{f}(\mathbf{k})= & 2 \sum_{\mathbf{p}} V_{\mathbf{p}}\left(\sin \frac{\mathbf{p} \times \mathbf{k}}{2}\right)^{2} \\
& \times\left[e^{-\mathbf{p} \cdot \mathbf{k}} \bar{s}(\mathbf{p}-\mathbf{k})-e^{-\frac{1}{2} \mathbf{k}^{2}} \bar{s}(\mathbf{p})\right]
\end{aligned}
$$

also starts with $O\left(\mathbf{k}^{4}\right)$,

$$
\bar{f}(\mathbf{k})=\frac{1}{2} \bar{\kappa}\left(\ell^{2} \mathbf{k}^{2}\right)^{2}+O\left(|\mathbf{k}|^{6}\right)
$$

so that the excitation has a gap $\epsilon_{\mathrm{SMA}}^{\text {coll }}=\bar{\kappa} / c$ at $\mathbf{k}=0$. The coefficient $\bar{\kappa}$ is given by

$\bar{\kappa}=\sum_{\mathbf{p}} V_{\mathbf{p}}\left[\frac{\mathbf{p}^{2}}{2}\left(D+\frac{1}{2}\right)+\frac{\left(\mathbf{p}^{2}\right)^{2}}{4}\left(D+\frac{1}{2}\right)^{2}\right] \bar{s}\left[\mathbf{p}^{2}\right]$

where $D=d / d\left(\mathbf{p}^{2}\right)$ acting on $\bar{s}(\mathbf{p})=\bar{s}\left[\mathbf{p}^{2}\right]$.

Let us now turn on $A_{\mu}$ and, as before, calculate terms that contribute to the $O\left(A^{2}\right)$ response eventually. See the Appendix for the evaluation of $i\left\langle\left[\theta \cdot \rho, \triangle H^{\text {Coul }}\right]\right\rangle$ and $\left\langle\left\{\lambda \cdot \rho, \triangle H^{\text {Coul }}\right\}\right\rangle$. The result is

$$
\mathcal{L}_{A}=\rho_{0}\left[-2 \lambda \bar{s} \chi-\bar{\kappa} \bar{\theta} \mathbf{k}^{2} \partial_{j} A_{j}+\bar{\kappa}^{\prime} \lambda \mathbf{k}^{2} A_{12}\right]
$$

to $O\left(\nabla^{3} A\right)$, where $\bar{\kappa}^{\prime}=\bar{\kappa}+(1 / 2) \sum_{\mathbf{p}} V_{\mathbf{p}}\left(\mathbf{p}^{2}\right)^{2} D^{2} \bar{s}\left[\mathbf{p}^{2}\right]$.

The effective Lagrangian $\mathcal{L}_{0}+\mathcal{L}_{A}$, governing the collective mode $\bar{\theta}$, takes essentially the same form as $\mathcal{L}_{A \theta}$ in Eq. (2.16). [The effect of $\bar{\kappa}^{\prime} \neq \bar{\kappa}$ is readily taken care of by the replacement $\delta \rightarrow\left(\bar{\kappa}^{\prime} / \bar{\kappa}\right)\left(1-s \omega_{c} / \bar{\kappa}^{\prime}\right)$ in the formulas of Sec. II, without spoiling gauge invariance $\left.{ }^{24}\right]$ With appropriate rescaling of the fields it is seen that the intra-Landau-level mode $\bar{\theta}$ behaves like a dipoleinactive $(0 \rightarrow 2)$ cyclotron mode in the electron system of Eq. (2.16) with $\omega_{c}=\hat{f} \rightarrow \frac{1}{2} \epsilon_{\text {SMA }}^{\text {coll }} \sim$ (Coulomb energy) at filling fraction $\nu_{\text {eff }}=4 \nu c=1-\nu$ (if we set $\hat{s}_{\text {eff }}=1$ ). Note that the collective mode disappears at $\nu=1$, as it should.

It is enlightening to compare the collective-mode spectrum with that in the composite-fermion (CF) theory. In the fermionic Chern-Simons theory of Lopez and Frad$\mathrm{kin},{ }^{9}$ the random-phase approximation (RPA) around the mean field for the Laughlin states with $\nu=1 / 3,1 / 5, \cdots$ gives rise to a family of collective modes with zeromomentum excitation gap $q \omega_{\mathrm{CF}}$ and static structure factors $\bar{s} \sim\left(\mathbf{k}^{2}\right)^{q}$, where $q=2,3, \cdots, 1 / \nu$, and $\omega_{\mathrm{CF}} \equiv$ $e B_{\text {eff }} / M_{\mathrm{CF}}=\nu \omega_{c}$ stands for the Landau gap for composite fermions. The mode with gap $\omega_{\mathrm{CF}}$ is missing and has been pushed up ${ }^{9.10}$ to the Landau gap $\omega_{c}$. The lowestlying collective mode, most stable among the family, thus has a gap $2 \omega_{\mathrm{CF}}$ and spectral weight $\bar{s} \sim\left(\mathbf{k}^{2}\right)^{2}$. Note that it has the same quadrupole character $\bar{s} \sim\left(\mathbf{k}^{2}\right)^{2}$ as the SMA collective mode. It is therefore natural to identify them and set

$$
\epsilon_{\mathrm{SMA}}^{\mathrm{coll}} \approx 2 \omega_{\mathrm{CF}} \text { for } \mathbf{k} \sim 0 .
$$

Let us here recall that in the CF theory, as discussed by Goldhaber and Jain ${ }^{25}$ the composite fermions themselves represent Laughlin's quasiparticles or vortices with fractional (renormalized) charge - $-\nu e$ (and bare charge $-e)$ and that $\omega_{\mathrm{CF}}$ is equal to the activation energy to create a widely-separated vortex-antivortex pair ${ }^{10.11}$ The spectrum (3.13) then suggests that the SMA collective mode at $\mathbf{k} \sim 0$ consists of four vortices (or a two-roton bound state ${ }^{13}$ with the roton regarded as a vortex-antivortex pair) in a quadrupole configuration so that $\bar{s} \sim\left(\mathbf{k}^{2}\right)^{2}$; this is in support of the Lee-Zhang picture $^{6}$ of the magnetoroton branch at $\mathbf{k} \sim 0$ within the composite-boson CS theory. This in turn gives, using the SMA value ${ }^{13}$ for $\epsilon_{\mathrm{SMA}}^{\text {coll }}$, the activation energy $\omega_{\mathrm{CF}}=\frac{1}{2} \epsilon_{\mathrm{SMA}}^{\text {coll }} \approx 0.075\left(e^{2} / 4 \pi \epsilon^{*} \ell\right)$ for the $\nu=1 / 3$ state, which is in rough agreement with other earlier estimates 13,14 Furthermore, for the $\nu=1 / 5$ state the SMA estimate yields $\frac{1}{2} \epsilon_{\mathrm{SMA}}^{\text {coll }} \approx 0.025\left(e^{2} / 4 \pi \epsilon^{*} \ell\right)$ so that $\left(\omega_{\mathrm{CF}}\right)_{\nu=1 / 5} /\left(\omega_{\mathrm{CF}}\right)_{\nu=1 / 3} \approx 1 / 3$, which is not far from the ratio $(3 / 5)^{2} \approx 1 / 2.8$ expected from the naive $\nu$ dependence of the activation energy.

The identification (3.13) has revealed nontrivial consistency among the SMA theory and CS theories, both bosonic and fermionic. We remark that this is a nonperturbative yet general result, in spite of the fact that in the CF theory corrections beyond the RPA affect ${ }^{9,10}$ the activation gap $\omega_{\mathrm{CF}}$ and the strength of higher-multipole 
responses substantially. Note that Eq. (3.13) essentially follows from the absence of a collective mode with a zero-momentum gap $\sim \omega_{\mathrm{CF}}$, which is generally the case (otherwise, the lowlying collective mode would become dipole-active, in violation of the f-sum rule). It would thus hold for the (exact) renormalized gap $\omega_{\mathrm{CF}}$ $\sim O\left(e^{2} / 4 \pi \epsilon^{*} \ell\right)$.

\section{BILAYER SYSTEMS}

In bilayer systems, unlike single-layer systems, some of intra-Landau-level excitations become dipole-active, and this makes the SMA and CS theories more distinct 17.19 In this section we construct an effective theory for bilayer systems. For clarity of discussion we consider systems without interlayer coherence and tunneling.

Consider a bilayer system with average electron densities $\rho_{0}^{[\alpha]}=\left(\rho_{0}^{[1]}, \rho_{0}^{[2]}\right)$ in the upper $(\alpha=1)$ and lower $(\alpha=2)$ layers. The system is placed in a common strong perpendicular magnetic field $B$ and, as before, we focus on the lowest Landau level $n=0$ (with the electron fields $\psi^{[\alpha]}$ in each layer taken to be fully spin polarized). The projected one-body Hamiltonian then reads

$$
\bar{H}_{1}=\sum_{\mathbf{p}}\left\{\chi_{\mathbf{p}}^{+} \bar{\rho}_{-\mathbf{p}}+\chi_{\mathbf{p}}^{-} \bar{d}_{-\mathbf{p}}\right\},
$$

where $\bar{\rho}_{\mathbf{p}}=\bar{\rho}_{\mathbf{p}}^{[1]}+\bar{\rho}_{\mathbf{p}}^{[2]}$ and $\bar{d}_{\mathbf{p}}=\bar{\rho}_{\mathbf{p}}^{[1]}-\bar{\rho}_{\mathbf{p}}^{[2]}$ are the projected charges; $\chi_{\mathbf{p}}^{ \pm}=\left(A_{0}^{ \pm}\right)_{\mathbf{p}}+(1 / 2 M)\left(A_{12}^{ \pm}\right)_{\mathbf{p}}$ and $A_{\mu}^{ \pm}(x)=\frac{1}{2}\left\{A_{\mu}^{[1]}(x) \pm A_{\mu}^{[2]}(x)\right\}$ in terms of weak external potentials $A_{\mu}^{[\alpha]}(x)$ acting on each layer.

The electrons in the two layers are coupled through the intralayer and interlayer Coulomb potentials $V_{\mathbf{p}}^{11}=V_{\mathbf{p}}^{22}$ and $V_{\mathbf{p}}^{12}=V_{\mathbf{p}}^{21}$, respectively; $V_{\mathbf{p}}^{11}=e^{2} /\left(2 \epsilon^{*}|\mathbf{p}|\right)$ and $V_{\mathbf{p}}^{12}=e^{-d|\mathbf{p}|} V_{\mathbf{p}}^{11}$ with the layer separation $d$ and the dielectric constant $\epsilon^{*}$ of the substrate. The projected Coulomb interaction is written $\mathrm{as}^{23}$

$$
\bar{H}^{\mathrm{C}}=\frac{1}{2} \sum_{\mathbf{p}}\left\{V_{\mathbf{p}}^{+} \bar{\rho}_{-\mathbf{p}} \bar{\rho}_{\mathbf{p}}+V_{\mathbf{p}}^{-} \bar{d}_{-\mathbf{p}} \bar{d}_{\mathbf{p}}\right\}+\triangle \bar{H}^{\mathrm{C}}
$$

with $V_{\mathbf{p}}^{ \pm}=\frac{1}{2}\left(V_{\mathbf{p}}^{11} \pm V_{\mathbf{p}}^{12}\right)$; the field-dependent piece $\triangle \bar{H}^{\mathrm{C}}$ takes essentially the same form as in the single-layer case.

There is a variety of quantum Hall states in bilayer systems $\stackrel{16.26}{1}$ For definiteness we consider bilayer quantum Hall states in a balanced configuration $\left(\rho_{0}^{[1]}=\rho_{0}^{[2]}\right)$, invariant under an interchange of the two layers. Of our particular concern are bilayer states with electron correlations, as described by Halperin's $(m, m, n)$ wave functions 26 at filling fractions $\nu=2 /(m+n)$; of these the $(3,3,1)$ state at $\nu=1 / 2$ has been observed experimentally 27

We here consider two types of collective excitations over such a bilayer state $|G\rangle$, the in-phase density excitations of the two layers, $\bar{\rho}_{\mathbf{k}}|G\rangle$ (probed by $A_{0}^{+}$), and the out-of-phase density excitations, $\bar{d}_{\mathbf{k}}|G\rangle$ (probed by $A_{0}^{-}$). Note that $\left\langle\bar{\rho}_{-\mathbf{k}} \bar{d}_{\mathbf{k}}\right\rangle=0$ for balanced configurations.
Kohn's theorem ${ }^{28}$ implies that the in-phase collective excitations remain dipole-inactive, as in the single-layer case, so that $\bar{s}_{+}(\mathbf{k}) \sim|\mathbf{k}|^{4}$ for small $\mathbf{k}$. On the other hand, interlayer interactions $V_{\mathbf{p}}^{12}$ spoils invariance under relative translations of the two layers and, unless interlayer coherence is realized, the out-of-phase collective excitations become dipole-active, $\stackrel{17.29}{,}$

$$
\bar{s}_{-}(\mathbf{k}) \equiv \frac{1}{N_{e}}\left\langle\bar{d}_{-\mathbf{k}} \bar{d}_{\mathbf{k}}\right\rangle=\hat{s}_{-} \frac{1}{2} \mathbf{k}^{2}+O\left(|\mathbf{k}|^{4}\right) .
$$

For the $(m, m, n)$ states the coefficient $\hat{s}_{-}$is given by ${ }^{29}$

$$
\hat{s}_{-}=2 n /(m-n) .
$$

To construct an effective theory let us denote the variations in phase and amplitude of the in-phase mode by $\theta$ and $\lambda$, and those of the out-of-phase mode by $\xi$ and $\eta$. Replacing $\theta \cdot \rho$ by $\theta \cdot \bar{\rho}+\xi \cdot \bar{d}$ and $\lambda \cdot \rho$ by $\lambda \cdot \bar{\rho}+\eta \cdot \bar{d}$ in the singlelayer expression (3.1) then yields an effective Lagrangian. The result splits into the $\left(\theta, \lambda, A_{\mu}^{+}\right)$and $\left(\xi, \eta, A_{\mu}^{-}\right)$sectors if one, as before, only retains terms contributing to the $O\left(A^{2}\right)$ response.

For the in-phase mode the effective theory is essentially the same as the single-layer case $\mathcal{L}_{0}+\mathcal{L}_{A}$ in Eqs. (3.6) and (3.12) with $A_{\mu} \rightarrow A_{\mu}^{+}, \bar{\kappa} \rightarrow \bar{\kappa}^{+}+\bar{\kappa}^{-}$and $\bar{\kappa}^{\prime} \rightarrow\left(\bar{\kappa}^{\prime}\right)^{+}+$ $\left(\bar{\kappa}^{\prime}\right)^{-}$. Here $\bar{\kappa}^{ \pm}$and $\left(\bar{\kappa}^{\prime}\right)^{ \pm}$are given by the corresponding single-layer expressions with $\left[V_{\mathbf{p}}^{ \pm}, \bar{s}^{ \pm}(\mathbf{p})\right]$.

For the out-of-phase mode the oscillator strength ${ }^{17,29}$ starts with $\mathbf{k}^{2}$,

$$
\begin{aligned}
& f_{-}(\mathbf{k})=\frac{1}{2} \kappa_{-} \mathbf{k}^{2}+O\left(|\mathbf{k}|^{4}\right), \\
& \kappa_{-}=2 \sum_{\mathbf{p}} \mathbf{p}^{2} V_{\mathbf{p}}^{12}\left\{-\bar{s}^{12}(\mathbf{p})\right\}
\end{aligned}
$$

where $\bar{s}^{12}(\mathbf{p}) \equiv\left\langle G\left|\rho_{-\mathbf{p}}^{[1]} \bar{\rho}_{\mathbf{p}}^{[2]}\right| G\right\rangle / N_{e}=\frac{1}{4}\left\{\bar{s}_{+}(\mathbf{p})-\bar{s}_{-}(\mathbf{p})\right\}$. This leads to the SMA excitation gap $\epsilon_{-}^{\text {coll }}=\kappa_{-} / \hat{s}_{-}$at $\mathbf{k} \rightarrow 0$. Eventually one is led to an effective Lagrangian of the form

$$
\begin{gathered}
\mathcal{L}_{-}^{\text {coll }}=\rho_{0}\left[2 \eta s_{-}\left(\dot{\xi}-\chi^{-}\right)-\left(\eta f_{-} \eta+\xi f_{-} \xi\right)\right. \\
\left.-\kappa_{-} \xi \partial_{j} A_{j}^{-}+\kappa_{-} \eta A_{12}^{-}\right]
\end{gathered}
$$

apart from terms of $O\left(\partial^{3} A^{-}\right)$. Again the coefficient $\kappa_{-}$of the $\xi \partial_{j} A_{j}^{-}$term is correlated with $f_{-}(\mathbf{k})$, in conformity with gauge invariance 24 With obvious substitution this $\mathcal{L}_{-}^{\text {coll }}$ is cast into the form of the effective Chern-Simons theory and dual-field theory of Eqs. (2.20) and (2.21), respectively, and leads to an out-of-phase response of the form of Eq. (2.23).

On the other hand, the $(0 \rightarrow 1)$ cyclotron modes $\xi^{[\alpha]}$ associated with each layer $\alpha=1,2$ are described by the effective CS theory of Eq. (2.20) with $\hat{s} \rightarrow 1$ and $\xi \rightarrow \xi^{[\alpha]}$, $A_{\mu} \rightarrow A_{\mu}^{[\alpha]}, \rho_{0} \rightarrow \rho_{0}^{[\alpha]}$, etc. The intra and interlayer Coulomb interactions are also correctly incorporated by use of an appropriate Hubbard-Stratonovich transformation. The effective theory agrees with the standard bilayer bosonic CS theory, ${ }^{30.31}$ except that the CS term has 
no interlayer mixing component. For the $(m, m, n)$ states the relevant mixing matrices (in terms of $\hat{s}$ ) differ by

$$
\left(\begin{array}{ll}
1 & 0 \\
0 & 1
\end{array}\right) \leftrightarrow \frac{1}{m-n}\left(\begin{array}{cc}
m & -n \\
-n & m
\end{array}\right) .
$$

The latter matrix in the CS theory is diagonalized in the $(\bar{\rho}, \bar{d})$ basis, yielding $\hat{s}_{+}^{\mathrm{CS}}=1$ (hence the correct Hall conductance $\left.\nu e^{2} / h\right)$ for the in-phase cyclotron mode and $\hat{s}_{-}^{\mathrm{CS}}=(m+n) /(m-n)$ for the out-of-phase mode.

It is important to note here that these dipole components of the structure factors govern the long-wavelength structure of many-body wave functions, as pointed out by Lopez and Fradkin ${ }^{32}$ This implies, in particular, that, if the quantum Hall state $|G\rangle$ embodies electronic correlations characteristic of the $(m, m, n)$ wave functions, one must have

$$
\hat{s}_{+}^{(m m n)}=1 \text { and } \hat{s}_{-}^{(m m n)}=(m+n) /(m-n) .
$$

This condition cannot be fulfilled by the cyclotron modes alone, which yield $\hat{s}_{ \pm}^{(10)}=1$, an inevitable consequence of the (projected) $f$-sum rule. This, in turn, implies the necessity of dipole-active intra-Landau-level excitations. Indeed, as seen form the SMA response (2.23), the cyclotron mode and collective mode combine to yield the desired out-of-phase $(m, m, n)$ electronic correlations

$$
1+\hat{s}_{-}=\frac{m+n}{m-n}=\hat{s}_{-}^{(m m n)} .
$$

On the other hand, the bilayer bosonic CS theory saturates the (out-of-phase) $f$-sum rule by a single dipoleactive mode so that $\hat{s}_{-}^{(m m n)} \omega_{\mathrm{CS}}^{\text {coll }} \approx \omega_{c}$. One thereby finds its spectrum at

$$
\omega_{\mathrm{CS}}^{\mathrm{coll}}=[(m-n) /(m+n)] \omega_{c}
$$

for $\mathbf{k} \rightarrow 0$. This unnatural shift of the out-of-phase cyclotron mode is attributed to the lack of projection in the bosonic CS theory (which thus fails to distinguish between the intra- and inter-Landau-level modes).

In the bilayer fermionic CS theory ${ }^{32}$ there emerge two dipole-active modes with strength $\frac{1}{2} \hat{s}_{-}^{(m m n)}$ at $\omega_{\mathrm{CS}}^{\text {coll }}$, thus resulting in essentially the same situation as in the bosonic CS theory. Apart from the collective-excitation spectrum, however, the SMA theory reproduces, owing to Eq. (4.9), the favorable long-wavelength transport properties of the CS theories, such as the Hall conductance, long-range orders, and fractional vortex charges.

A good place to detect the out-of-phase collective mode would be Hall drag experiments ${ }^{33}$ The interlayer Hall conductance becomes sizable ${ }^{34}$ in the presence of the $(m, m, n)$ correlations, as read from the electromagnetic response (2.23):

$$
\left(\begin{array}{c}
J_{x}^{[1]} \\
J_{x}^{[2]}
\end{array}\right)=-\frac{e^{2} \nu}{4 h}\left(\begin{array}{cc}
\sigma^{+}+\sigma^{-} & \sigma^{+}-\sigma^{-} \\
\sigma^{+}-\sigma^{-} & \sigma^{+}+\sigma^{-}
\end{array}\right)\left(\begin{array}{c}
E_{y}^{[1]} \\
E_{y}^{[2]}
\end{array}\right),
$$

where $\sigma^{+}=1 /\left[1-\left(\omega / \omega_{c}\right)^{2}\right]$ and $\sigma^{-}=1 /\left[1-\left(\omega / \omega_{c}\right)^{2}\right]+$ $\hat{s}_{-} /\left[1-\left(\omega / \epsilon_{-}^{\text {coll }}\right)^{2}\right]$. For the $(3,3,1)$ state a direct current $J_{x}^{[1]}$ injected to the upper layer would induce a Hall voltage $V_{y}^{[2]}=\left(h / e^{2}\right) J_{x}^{[1]}$ on the lower layer (left to be an open circuit) while yielding $V_{y}^{[1]}=3\left(h / e^{2}\right) J_{x}^{[1]}$ on the same layer. The interlayer resistivity is sensitive to the collective mode through its response to time-varying currents,

$$
\rho_{y x}^{[21]} \approx-\left(h / e^{2}\right) n /\left[1-\beta \omega^{2} /\left(\epsilon_{-}^{\text {coll }}\right)^{2}\right]
$$

with $\beta=(m-n) /(m+n)$ while $\rho_{y x}^{[11]}+\rho_{y x}^{[21]} \approx$ $-\left(h / e^{2}\right)(m+n)$ stays fixed for $\omega \leq \epsilon_{-}^{\text {coll }} \ll \omega_{c}$. Since the quantized Hall resistance (for $\omega=0$ ) is expected to be very accurate, there would be a good chance of detecting such a difference in experiments with an injected high-frequency current or a current pulse.

\section{SUMMARY AND DISCUSSION}

In this paper we have presented a unified treatment of elementary and collective excitations in quantum Hall systems by means of the single-mode approximation (SMA). A variational principle and nonlinear realizations of the $W_{\infty}$ algebra have been combined to construct effective theories that incorporate the SMA excitation spectrum. For a wide class of quantum Hall systems the resulting effective theory turns out to be a variant of the bosonic CS theory. In this sense, there is a direct link between the composite-boson theory and the SMA treatment of quantum Hall systems. The CS action therefore is a quite natural element for quantum Hall systems, irrespective of the notion of flux attachment.

We have noted that the intra-Landau-level modes bear a similarity in structure (though not in scale) to the interLandau-level modes. This has revealed a further link with CS theories: A comparison with the fermionic CS theory suggests that the SMA collective modes in singlelayer systems (around $\mathbf{k} \approx 0$ ) are composed of four vortices in a quadrupole configuration, in conformity with an interpretation $\underline{\underline{\underline{6}}}$ within the bosonic CS theory.

In the SMA all the information is essentially contained in the structure factors $s(\mathbf{k})$, which represent electronic correlations pertinent to the quantum Hall state in question. In particular, the dipole part $\propto \mathbf{k}^{2}$ of $s(\mathbf{k})$ is directly related to the long-wavelength structure of many-body wave functions and also to the flux attachment transformation employed in CS approaches. This fact has some important consequences. First, for single-layer systems such $O\left(\mathbf{k}^{2}\right)$ correlations are governed by the cyclotron modes alone (as implied by Kohn's theorem), so is the CS-flux attachment. This resolves the puzzle why the main features of the single-layer CS theories, such as the Hall conductance, long-range orders and fractional charges of quasiparticles, are apparently determined by the cyclotron modes (or the electronic kinetic term which one would naively expect to be quenched) while the 
FQHE is actually caused by the Coulomb interaction. In this sense, the dipole correlation with $\hat{s}^{(10)}=1$ is characteristic of typical single-layer quantum Hall states.

For bilayer systems the situation is different. Correlations characteristic of the bilayer $(m, m, n)$ states, for example, are not attained by the cyclotron modes alone and require the presence of dipole-active collective modes. As a result, the SMA effective theory is bound to involve such collective modes and deviates from a naive bilayer version of the bosonic CS theory.

While the structure factors $s(\mathbf{k})$ are readily calculated for the cyclotron modes via projection, the determination of $s(\mathbf{k})$ for collective modes is a nontrivial subject of dynamics, handled in a variety of approximation schemes. In the present paper we have simply focused on quantum Hall states well approximated by Laughlin's or Halperin's wave functions. There is a practical way to improve the structure factors. One may appeal to Jain's compositefermion wave-function approach,,$\frac{4}{-}$ which is known to yield numerically very accurate variational wave functions and which, along with refinement to incorporate corrections due to layer thickness, Coulombic Landau-level mixing, etc., has been generalized to bilayer systems as well ${ }^{35}$ With such improved structure factors one could refine the effective theory and make comparisons with experiments more reliable.

\section{Acknowledgments}

This work was supported in part by a Grant-in-Aid for Scientific Research from the Ministry of Education of
Japan, Science and Culture (Grant No. 14540261).

\section{APPENDIX A: CALCULATION}

In this appendix we outline the calculation of the electromagnetic coupling (3.12) coming from the fielddependent Coulomb interaction $\triangle H^{\text {Coul }}$. Consider a factor $\Lambda_{\mathbf{k}, \mathbf{p}}=\left(1 / 2 N_{e}\right)\left\langle G\left|\bar{\rho}_{\mathbf{k}}\left\{\bar{\rho}_{-\mathbf{p}}, \bar{\rho}_{\mathbf{p}-\mathbf{k}}\right\}\right| G\right\rangle$ involving products of three projected charges. As discussed in an earlier SMA treatment $\stackrel{19}{=}$ the leading small $\mathbf{k}$ behavior of such products is determined from the portion that originates from the noncommutative nature $\left[r_{1}, r_{2}\right]=i \ell^{2}$ of $\mathbf{r}$, with the result

$$
\begin{aligned}
\Lambda_{\mathbf{k}, \mathbf{p}}= & \left(e^{-\frac{1}{2} k^{\dagger} p}-1\right) \bar{s}(\mathbf{p}-\mathbf{k})+\left(e^{\frac{1}{2} k^{\dagger}(p-k)}-1\right) \bar{s}(\mathbf{p}) \\
& +O\left(\mathbf{k}^{4}\right),
\end{aligned}
$$

where $k^{\dagger} p=\mathbf{k} \cdot \mathbf{p}-i \mathbf{k} \times \mathbf{p}$. The factors relevant for the $\left\langle\left\{\lambda \cdot \rho, \triangle H^{\text {Coul }}\right\}\right\rangle$ and $i\left\langle\left[\theta \cdot \rho, \triangle H^{\text {Coul }}\right]\right\rangle$ terms are then given by the real and imaginary part of $\Lambda_{\mathbf{k}, \mathbf{p}}$, respectively. Note, as an independent check, that $\left[\theta \cdot \rho, \triangle H^{\mathrm{Coul}}\right]$ is also determined from the charge algebra (2.4) alone, yielding the same result.
1 D. C. Tsui, H. L. Stormer, and A. C. Gossard, Phys. Rev. Lett. 48, 1559 (1982).

2 R. B. Laughlin, Phys. Rev. Lett. 50, 1395 (1983).

3 S. M. Girvin and A. H. MacDonald, Phys. Rev. Lett. 58, 1252 (1987); N. Read, Phys. Rev. Lett. 62, 86 (1989).

4 J. K. Jain, Phys. Rev. Lett. 63, 199 (1989).

5 S. C. Zhang, T.H. Hansson, and S. Kivelson, Phys. Rev. Lett. 62, 82 (1989).

6 D.-H. Lee and S.-C. Zhang, Phys. Rev. Lett. 66, 1220 (1991).

7 S. C. Zhang, Int. J. Mod. Phys. B 6, 25 (1992).

8 B. Blok and X. G. Wen, Phys. Rev. B 42, 8133 (1990).

9 A. Lopez and E. Fradkin, Phys. Rev. B 44, 5246 (1991); ibid. 47, 7080 (1993).

10 B. I. Halperin, P. A. Lee, and N. Read, Phys. Rev. B 47, 7312 (1993).

11 C. Kallin and B. I. Halperin, Phys. Rev. B 30, 30, 5655 (1984).

12 S. M. Girvin and T. Jach, Phys. Rev. B 29, 5617 (1984).

13 S. M. Girvin, A. H. MacDonald, and P. M. Platzman, Phys. Rev. Lett. 54, 581 (1985); Phys. Rev. B 33, 2481 (1986).

14 F. D. M. Haldane and E. H. Rezayi, Phys. Rev. Lett. 54, 237 (1985).

15 A. H. MacDonald, H. C. A. Oji, and S. M. Girvin, Phys.
Rev. Lett. 55, 2208 (1985).

16 T. Chakraborty and P. Pietilainen, Phys. Rev. Lett. 59, 2784 (1987); D. Yoshioka, A. H. MacDonald, and S. M. Girvin, Phys. Rev. B 39, 1932 (1989); H. A. Fertig, Phys. Rev. B 40, 1087 (1989).

17 A. H. MacDonald and S.-C. Zhang, Phys. Rev. B 49, 17 208 (1994).

18 K. Moon, H. Mori, K. Yang, S. M. Girvin, A. H. MacDonald, L. Zheng, D. Yoshioka, and S.-C. Zhang, Phys. Rev. B 51, 5138 (1995).

19 K. Shizuya, Phys. Rev. B 65, 205324 (2002); ibid. 63, 245301 (2001).

${ }^{20}$ K. Shizuya, Phys. Rev. B 45, 11143 (1992).

21 M. V. Berry, Proc. R. Soc. London, Ser. A 392, 45 (1984).

22 A. Pinczuk, B. S. Dennis, L. N. Pfeiffer, and K. West, Phys. Rev. Lett. 70, 3983 (1993).

23 To be precise, $\bar{\rho}_{\mathbf{p}}$ in $\bar{H}^{\text {Coul }}$ refers to deviations $\bar{\rho}_{\mathbf{p}}-\rho_{0}$ from the background charge; analogously for $\bar{\rho}_{\mathbf{p}}$ and $\bar{d}_{\mathbf{p}}$ in $\bar{H}^{\mathrm{C}}$.

24 Also a direct calculation shows that a gauge-variant $O\left(A^{2}\right)$ term following from Eq. (3.12) is canceled by a term coming from the field-dependent Coulomb interaction of $O\left(A^{2}\right)$; analogously for Eq. (4.6).

25 A. S. Goldhaber and J. K. Jain, Phys. Lett. A 199, 267 (1995); for the renormalized (fractional) charge of compos- 
ite fermions see also the second reference in Ref 19 .

26 B. I. Halperin, Helv. Phys. Acta 56, 75 (1983).

27 Y. W. Suen, L. W. Engel, M. B. Santos, M. Shayegan, and D. C. Tsui, Phys. Rev. Lett. 68, 1379 (1992); J. P. Eisenstein, G. S. Boebinger, L. N. Pfeiffer, K. W. West, and S. He, Phys. Rev. Lett. 68, 1383 (1992).

${ }^{28}$ W. Kohn, Phys. Rev. 123, 1242 (1961).

29 S. R. Renn and B. W. Roberts, Phys. Rev. B 48, 10926 (1993).

30 X. G. Wen and A. Zee, Phys. Rev. Lett. 69, 1811 (1992).

31 Z. F. Ezawa and A. Iwazaki, Int. J. Mod. Phys. B 6, 3205
(1992).

32 A. Lopez and E. Fradkin, Phys. Rev. B 51, 4347 (1995). (bilayers)

33 M. Kellogg, I. B. Spielman, J. P. Eisenstein, L. N. Pfeiffer, and K. W. West, Phys. Rev. Lett. 88, 126804 (2002).

34 S. R. Renn, Phys. Rev. Lett. 68, 658 (1992); K. Yang, Phys. Rev. B 58, R4246 (1998).

35 V. W. Scarola, K. Park, and J. K. Jain, Phys. Rev. B 61, 13064 (2000); V. W. Scarola and J. K. Jain, Phys. Rev. B 64, 085313 (2001). 